\title{
Exponential Attractor for the Boussinesq Equation with Strong Damping and Clamped Boundary Condition
}

\author{
Fan Geng, ${ }^{1}$ Ruizhai Li, ${ }^{1}$ Xiaojun Zhang, ${ }^{2}$ and Xiangyu Ge ${ }^{2}$ \\ ${ }^{1}$ School of Arts and Science, Sias International University, Zhengzhou 451150, China \\ ${ }^{2}$ Wuhan Technology and Business University, Wuhan 430065, China \\ Correspondence should be addressed to Xiangyu Ge; xiangyu_ge@163.com
}

Received 29 November 2015; Accepted 7 February 2016

Academic Editor: Taher S. Hassan

Copyright (c) 2016 Fan Geng et al. This is an open access article distributed under the Creative Commons Attribution License, which permits unrestricted use, distribution, and reproduction in any medium, provided the original work is properly cited.

The paper studies the existence of exponential attractor for the Boussinesq equation with strong damping and clamped boundary condition $u_{t t}-\Delta u+\Delta^{2} u-\Delta u_{t}-\Delta g(u)=f(x)$. The main result is concerned with nonlinearities $g(u)$ with supercritical growth. In that case, we construct a bounded absorbing set with further regularity and obtain quasi-stability estimates. Then the exponential attractor is established in natural energy space $V_{2} \times H$.

\section{Introduction}

In this paper, we are concerned with the existence of exponential attractor for the Boussinesq equation with strong damping and clamped boundary condition

$$
u_{t t}-\Delta u+\Delta^{2} u-\Delta u_{t}-\Delta g(u)=f(x) \quad \text { in } \Omega \times \mathbb{R}^{+},
$$

where $\Omega$ is a bounded domain in $\mathbb{R}^{N}$ with the smooth boundary $\partial \Omega$, on which we consider the clamped boundary condition

$$
\begin{aligned}
\left.u\right|_{\partial \Omega} & =0, \\
\left.\frac{\partial u}{\partial \nu}\right|_{\partial \Omega} & =0,
\end{aligned}
$$

where $v$ is the unit outward normal on $\partial \Omega$, and the initial condition

$$
\begin{gathered}
u(x, 0)=u_{0}(x), \\
u_{t}(x, 0)=u_{1}(x),
\end{gathered}
$$

$$
x \in \Omega,
$$

and the assumptions on $g(u)$ and $f$ will be specified later.

In 1872, Boussinesq [1] established the equation

$$
u_{t t}-u_{x x}-\alpha u_{x x x x}=\beta\left(u^{2}\right)_{x x}
$$

to describe the longitudinal displacement of the shallow water wave. Here $u$ and $\alpha, \beta$ are some constants depending on the depth of the fluid and characteristic velocity of the water wave. When $\alpha<0$, (4) is called "good" Boussinesq equation, when $\alpha>0$, (4) is called "bad" Boussinesq equation. There have been lots of research on the well-posedness, blowup, and other properties of solutions for both the "good" and the "bad" Boussinesq equation of type (1) (see [2-14] and references therein). While for the investigation on the global attractor to (1), one can see [15-19] and references therein.

Chueshov and Lasiecka [20, 21] studied the longtime behavior of solutions to the Kirchhoff-Boussinesq plate equation

$$
u_{t t}+k u_{t}+\Delta^{2} u=\operatorname{div}\left[f_{0}(\nabla u)\right]+\Delta\left[f_{1}(u)\right]-f_{2}(u)
$$

with $\Omega \subset \mathbb{R}^{2}$ and the clamped boundary condition (2). Here $k>0$ is the damping parameter and the mapping $f_{0}: \mathbb{R}^{2} \rightarrow$ $\mathbb{R}^{2}$ and the smooth functions $f_{1}$ and $f_{2}$ represent (nonlinear) feedback forces acting upon the plate, in particular,

$$
\begin{gathered}
f_{0}(\nabla u)=|\nabla u|^{2} \nabla u, \\
f_{1}(u)=u^{2}+u .
\end{gathered}
$$

Ignoring both restoring force $f_{0}(\nabla u)$ and feedback force $f_{2}(u)$ and replacing the inertial term $u_{t t}$ by $\epsilon u_{t t}$, with $\epsilon>0$ (the 
relaxation time) sufficiently small, (5) becomes the modified Cohn-Hilliard equation

$$
\epsilon u_{t t}+u_{t}-\Delta(-\Delta u+f(u))=g,
$$

which is proposed by Galenko et al. [22-24] to model rapid spinodal decomposition in nonequilibrium phase separation processes. Grasselli et al. [25-27] studied the well-posedness and the longtime dynamics of (7) in both $2 D$ and $3 D$ cases, with hinged boundary condition. They established the existence of the global and exponential attractor for $\epsilon=1$ in $2 D$ case, and for $\epsilon>0$ sufficiently small in $3 D$ case. Taking $\epsilon=1$ in (7) or taking $f_{0}(\nabla u)=\nabla u, f_{2}=0$ in (5), and taking into account the inertial force represented by $-\Delta u$ and replacing the weak damping $u_{t}$ by a strong one $-\Delta u_{t}$, (1) arises.

In $1 D$ case, Dai and Guo $[15,16]$ studied the "bad" Boussinesq equation with strong damping

$$
\begin{aligned}
u_{t t}-u_{x x}+2 k u_{x x t}-\alpha u_{x x x x} & =\beta\left(u^{n}\right)_{x x} \\
\quad \text { in } \mathbb{R} \times(0,+\infty), & \\
u(x, 0) & =u_{0}(x), \\
u_{t}(x, 0) & =u_{1}(x),
\end{aligned}
$$

$$
x \in \mathbb{R} .
$$

They got global solution $u \in C^{\infty}\left((0, T] ; H^{\infty}(R)\right) \cap C([0, T]$; $\left.H^{1}(R)\right) \cap C\left([0, T] ; H^{-1}(R)\right) \forall T>0$, where $k, \alpha \in \mathbb{R}^{+}, \beta \in \mathbb{R}^{+}$, $n>2$.

For the multidimensional case, Yang [17] proved the IVP of the Boussinesq equation

$$
\begin{aligned}
u_{t t}-\Delta u+\mu \Delta^{2} u & =\Delta \sigma(u) \quad \text { in } \mathbb{R}^{N} \times(0,+\infty), \\
u(x, 0) & =u_{0}(x), \\
u_{t}(x, 0) & =u_{1}(x), \\
x \in R^{N} . &
\end{aligned}
$$

There existed the global weak solution, where $\mu>0, \sigma(u) \epsilon$ $C(R),|\sigma(s)| \leq b|s|^{p}, s \in R, 1<p \leq(N+2) /(N-2)^{+}$, and $0 \leq \sigma(s) s \leq \beta \int_{0}^{s} \sigma(\tau) d \tau, s \in \mathbb{R}, \beta>0$. Here the growth exponent $\tilde{p}=N /(N-2)(N \geq 3)$ is called critical. The growth exponent $p^{*} \equiv(N+2) /(N-2)^{+}(\geq \widetilde{p})$ is called supercritical. However, there is little research on the the higher global regularity of a bounded absorbing set, the global attractor and an exponential attractor in natural energy space for the dynamical system. We try to solve those problems in this paper.

Global attractor is a basic concept in the research studies of the asymptotic behavior of the dissipative system. From the physical point of view, the global attractor of the dissipative equation (1) represents the permanent regime that can be observed when the excitation starts from any point in natural energy space, and its dimension represents the number of degrees of freedom of the related turbulent phenomenon and thus the level of complexity concerning the flow. All the information concerning the attractor and its dimension from the qualitative nature to the quantitative nature then yields valuable information concerning the flows that this physical system can generate. On the physical and numerical sides, this dimension gives one an idea of the number of parameters and the size of the computations needed in numerical simulations. However, the global attractor may possess an essential drawback; namely, the rate of attraction may be arbitrarily slow and it can not be estimated in terms of physical parameters of the system under consideration. While the exponential attractor overcomes the drawback because not only it has finite fractal dimension but also its contractive rate is exponential and measurable in terms of the physical parameters, the purpose of the present paper is to establish the existence of an exponential attractor in supercritical case. Our result (see Theorem 8 below) in this paper extends the corresponding result in [28].

In comparison with the results in $[17,18]$, the contribution of the paper lies in that

(1) the exponential attractor is established in natural energy space $E$ in supercritical case. See Theorem 8;

(2) the critical case $p=\widetilde{p}$ is solved in $E_{1}$. In the concrete, when $1 \leq p \leq \widetilde{p}$, the global and exponential attractor in $E_{1}$ is established, and the higher regularity of the global attractor is obtained. See Theorem 15;

(3) the restriction $N \leq 5$ is removed in subcritical case. See Theorem 15.

The plan of the paper is as follows. In Section 2, the global existence of the weak solutions is discussed by the energy method and the existence of global attractor is established. In Section 3, the exponential attractor is established for supercritical case. In Section 4, global attractor and the exponential attractor are established for nonsupercritical case.

\section{Global Existence of Weak Solutions}

For brevity, we use the following abbreviations:

$$
\begin{aligned}
L^{p} & =L^{p}(\Omega), \\
H^{k} & =H^{k}(\Omega), \\
H & =L^{2}, \\
V_{2} & =H_{0}^{2}, \\
\|\cdot\| & =\|\cdot\|_{L^{2}}, \\
\|\cdot\|_{p} & =\|\cdot\|_{L^{p}},
\end{aligned}
$$

with $p \geq 1$, where $H^{k}$ are the $L^{2}$-based Sobolev spaces and $H_{0}^{k}$ are the completion of $C_{0}^{\infty}(\Omega)$ in $H^{k}$ for $k>0$. The notation $(\cdot, \cdot)$ for the $H$-inner product will also be used for the notation of duality pairing between dual spaces and $C(\cdots)$ denotes positive constants depending on the quantities appearing in the parenthesis. $\left.V_{2}\right)$,

We define the operator $A: V_{2} \rightarrow V_{2}^{\prime}$ (the dual space of

$$
(A u, v)=(\Delta u, \Delta v) \quad \text { for any } u, v \in V_{2} \text {. }
$$


Then, the operators $A^{s}(s \in \mathbb{R})$ are strictly positive and the spaces $V_{s}=D\left(A^{s / 4}\right)$ are Hilbert spaces with the scalar products and the norms

$$
\begin{aligned}
(u, v)_{s} & =\left(A^{s / 4} u, A^{s / 4} v\right), \\
\|u\|_{V_{s}} & =\left\|A^{s / 4} u\right\|,
\end{aligned}
$$

respectively. Obviously,

$$
\begin{aligned}
& \|u\|_{V_{2}}=\left\|A^{1 / 2} u\right\|=\|\Delta u\|, \\
& \|u\|_{V_{1}}=\left\|A^{1 / 4} u\right\|=\|\nabla u\| .
\end{aligned}
$$

Rewriting (1) in the operator equation and applying $A^{-1 / 2}$ to the resulting expression, we get the Cauchy problem equivalent to problem (1), (2), and (3):

$$
\begin{aligned}
A^{-1 / 2} u_{t t}+\left(I+A^{1 / 2}\right) u+u_{t}+g(u) & =A^{-1 / 2} f, \\
u(0) & =u_{0}, \\
u_{t}(0) & =u_{1} .
\end{aligned}
$$

For each $s \in \mathbb{R}, V_{s}=D\left(A^{s / 4}\right)$, we denote the Banach space

$$
\begin{aligned}
E & =V_{2} \times H, \\
E_{1} & =V_{1} \times H,
\end{aligned}
$$

which is equipped with the usual graph norm,

$$
\|(u, v)\|_{E}^{2}=\|u\|_{V_{2}}^{2}+\|v\|^{2} .
$$

Theorem 1. Assume that $\left(H_{1}\right) g \in C^{1}(\mathbb{R})$,

$$
\begin{aligned}
& |g(s)| \leq K_{1}\left(|s|^{p}+1\right), \\
& \left|g^{\prime}(s)\right| \leq K_{2}\left(|s|^{p-1}+1\right), \\
& \left|g^{\prime}\left(s_{1}\right)-g^{\prime}\left(s_{2}\right)\right| \\
& \quad \leq K_{3}\left(\left|s_{1}\right|^{p-1-\gamma}+\left|s_{2}\right|^{p-1-\gamma}+1\right)\left|s_{1}-s_{2}\right|^{\gamma},
\end{aligned}
$$

where

$$
\begin{aligned}
& 0<\gamma<p-1, \quad 1<p<2, \\
& \gamma=1, \quad p \geq 2,
\end{aligned}
$$

where $(a)^{+}=\max \{0, a\}, K_{i}>0, i=1,2,3,1 \leq p \leq p^{*} \equiv$ $(N+2) /(N-2)$, and $N \geq 3$.

$\left(\mathrm{H}_{2}\right)$ Consider

$$
\begin{array}{r}
\liminf _{|s| \rightarrow+\infty} \frac{G(s)}{|s|^{2}} \geq 0, \\
\liminf _{|s| \rightarrow+\infty} \frac{s g(s)-\rho G(s)}{|s|^{2}} \geq 0,
\end{array}
$$

where $G(s)=\int_{0}^{s} g(\tau) d \tau, 0<\rho<2$.

$\left(H_{3}\right)\left(u_{0}, u_{1}\right) \in E, f \in V_{-1}$.

Then problem (14) and (15) admits a unique weak solution $u$, with $\left(u, u_{t}\right) \in C_{b}\left(R^{+}, V_{2} \times H\right)$. More precisely, the solution $u$ possesses the following properties: (i) There exists a small positive constant $\delta$ such that

$$
\begin{aligned}
& \left\|\left(u, u_{t}\right)\right\|_{E}^{2}+\int_{t}^{t+1}\left\|u_{t}(\tau)\right\|^{2} d \tau \\
& \quad \leq C\left(\left\|\left(u_{0}, u_{1}\right)\right\|_{E}\right) e^{-\delta t}+C\left(\|f\|_{V_{-1}}\right), \quad t \geq 0 .
\end{aligned}
$$

(ii) When $1 \leq p<p^{*}$, the solution is Lipschitz continuous in the weaker space $E$ as $N \leq 5$; that is,

$$
\left\|\left(z(t), z_{t}(t)\right)\right\|_{E}^{2} \leq C(R) e^{k t}\left\|\left(z(0), z_{t}(0)\right)\right\|_{E}^{2},
$$

$$
t \geq 0 \text {, }
$$

for some $k>0$, where $z=u-v, u$ and $v$ are, respectively, the weak solutions of (14) corresponding to initial data $\left(u_{0}, u_{1}\right)$ and $\left(v_{0}, v_{1}\right)$.

Remark 2. The formula (20) implies that every $\eta>0$; there exists $C_{\eta}>0, \widetilde{C}_{\eta}>0$ such that

$$
\begin{array}{r}
G(s)+\eta|s|^{2} \geq-C_{\eta}, \\
s g(s)-\rho G(s)+\eta|s|^{2} \geq-\widetilde{C}_{\eta},
\end{array}
$$

where $G(s)=\int_{0}^{s} g(\tau) d \tau$.

Lemma 3 (see [29]). Let $X, B$, and $Y$ be the Banach spaces, $X \hookrightarrow \hookrightarrow B \hookrightarrow Y$,

$$
\begin{gathered}
W=\left\{u \in L^{p}(0, T ; X) \mid u_{t} \in L^{1}(0, T ; Y)\right\}, \\
\text { with } 1 \leq p<\infty, \\
W_{1}=\left\{u \in L^{\infty}(0, T ; X) \mid u_{t} \in L^{r}(0, T ; Y)\right\}, \\
\text { with } r>1 .
\end{gathered}
$$

Then,

$$
\begin{gathered}
W \hookrightarrow \hookrightarrow L^{p}(0, T ; B), \\
W_{1} \hookrightarrow \hookrightarrow C([0, T] ; B) .
\end{gathered}
$$

Proof of Theorem 1. We first obtain a priori estimate to the solutions of problem (14) and (15).

Let $v=u_{t}+\epsilon u$ and rewrite (1); we have

$$
\begin{aligned}
& A^{-1 / 2}\left(v_{t}-\epsilon v+\epsilon^{2} u\right)+\left(I+A^{1 / 2}\right) u+v-\epsilon u+g(u) \\
& \quad=A^{-1 / 2} f \\
& v(0)=u_{1}+\epsilon u_{0}=v_{0} .
\end{aligned}
$$

Using the multiplier $u_{t}$ in (26), we get

$$
\frac{d}{d t} H_{1}(u, v)+K_{1}(u, v)=0, \quad t>0,
$$


where

$$
\begin{aligned}
& H_{1}\left(u, u_{t}\right)=\frac{1}{2}\left(\left\|A^{-1 / 4} v\right\|^{2}+\epsilon^{2}\left\|A^{-1 / 4} u\right\|^{2}+\left\|A^{1 / 4} u\right\|^{2}\right. \\
& \left.+(1-\epsilon)\|u\|^{2}+2 \int_{\Omega} G(u) d x-2\left(A^{-1 / 2} f, u\right)\right) \\
& \quad \geq C_{1}\left(\left\|A^{-1 / 4} u_{t}\right\|^{2}+\left\|A^{-1 / 4} u\right\|^{2}+\left\|A^{1 / 4} u\right\|^{2}+\|u\|^{2}\right) \\
& \quad-C_{0}, \\
& K_{1}(u, v)-\epsilon H_{1}(u, v) \geq C\left(\|v\|^{2}+\left\|A^{-1 / 4} v\right\|^{2}\right. \\
& \left.\quad+\left\|A^{1 / 4} u\right\|^{2}\right)-C_{0} .
\end{aligned}
$$

Hence,

$$
\frac{d}{d t} H_{1}(u, v)+\epsilon H_{1}(u, v) \leq-C_{0}, \quad t \geq 0 .
$$

Applying the Gronwall lemma to (29),

$$
\left\|A^{1 / 4} u\right\|^{2}+\left\|A^{-1 / 4} u_{t}\right\|^{2} \leq C_{1} e^{-\delta t}+C_{0}
$$

where $C_{0}=\left(\|f\|_{V_{-1}}\right), C_{1}=\left(\left\|\left(u_{0}, u_{1}\right)\right\|_{E_{1}}\right), \delta=\epsilon \in(0,1)$.

Using the multiplier $A^{1 / 2} u_{t}, A^{1 / 2} u$ in (26),

$$
\begin{aligned}
& \frac{1}{2} \frac{d}{d t}\left(\left\|u_{t}\right\|^{2}+\left\|A^{1 / 4} u\right\|^{2}+\left\|A^{1 / 2} u\right\|^{2}-2(u, f)\right) \\
& \quad+\left\|A^{1 / 4} u_{t}\right\|^{2}+\left(A^{1 / 2} u_{t}, g(u)\right)=0, \quad t>0, \\
& \frac{d}{d t}\left(\frac{1}{2}\left\|A^{1 / 4} u\right\|^{2}+\left(u_{t}, u\right)\right)+\left\|A^{1 / 4} u\right\|^{2} \\
& \quad+\left(A^{1 / 2} u, g(u)\right)=\left\|u_{t}\right\|^{2}+(u, f), \quad t>0,
\end{aligned}
$$

$(31)+\epsilon(32)$; we have

$$
\frac{d}{d t} H_{2}(u)+K_{2}(u)=0, \quad t>0 .
$$

Obviously,

$$
\begin{aligned}
H_{2}(u)= & \frac{1}{2}\left[\left\|u_{t}\right\|^{2}+(1+\epsilon)\left\|A^{1 / 4} u\right\|^{2}+\left\|A^{1 / 2} u\right\|^{2}\right] \\
& +\epsilon\left(u, u_{t}\right)-(u, f) \\
\sim & \left\|A^{1 / 4} u\right\|^{2}+\left\|u_{t}\right\|^{2}+\left\|A^{1 / 2} u\right\|^{2}, \\
K_{2}(u)= & \left\|A^{1 / 4} u_{t}\right\|^{2}-\epsilon\left\|u_{t}\right\|^{2}+\left(A^{1 / 4} g(u), A^{1 / 4} u_{t}\right) \\
& +\epsilon\left(\left\|A^{1 / 2} u\right\|^{2}+\left(A^{1 / 2} u, g(u)\right)-(u, f)\right) \\
\geq & C\left(\left\|A^{1 / 4} u_{t}\right\|^{2}+\left\|A^{1 / 2} u\right\|^{2}\right)-C_{1} e^{-\delta t}-C_{0} .
\end{aligned}
$$

Indeed,

$$
\begin{aligned}
\left|\left(A^{1 / 4} g(u), A^{1 / 4} u_{t}\right)\right| \leq & \frac{1}{4}\left\|A^{1 / 4} u_{t}\right\|^{2}+\frac{\varepsilon}{4}\left\|A^{1 / 2} u\right\|^{2} \\
& +C, \\
\left|\left(A^{1 / 2} u, g(u)\right)\right| \leq & \frac{1}{4}\left\|A^{1 / 2} u\right\|^{2}+C,
\end{aligned}
$$

where $1 \leq p<N /(N-2)^{+}, H^{s} \hookrightarrow L^{2 p} ; N /(N-2)^{+} \leq p<$ $(N+2) /(N-2)^{+}, V_{2-\delta} \hookrightarrow L^{2(p+1)}$, and $H^{1} \hookrightarrow L^{p+1}$

$$
\frac{d}{d t} H_{2}(u)+\delta H_{2}(u)+\delta\left\|A^{1 / 4} u_{t}(t)\right\|^{2} \leq C_{1} e^{-\delta t}+C_{0} .
$$

Thus

$$
\left\|A^{1 / 2} u\right\|^{2}+\left\|A^{1 / 4} u\right\|^{2}+\left\|u_{t}\right\|^{2} \leq C_{2} e^{-\delta t}+C_{0}, \quad t>0 .
$$

That is,

$$
\left\|\left(u, u_{t}\right)\right\|_{E}+\int_{t}^{t+1}\left\|A^{1 / 4} u_{t}(\tau)\right\|^{2} d \tau \leq C_{2} e^{-\delta t}+C_{0},
$$

$$
t>0 \text {. }
$$

It follows from (14) and (38) that

$$
\begin{aligned}
u_{t t} & =f-A^{1 / 2} u-A^{1 / 2} u_{t}-A u-A^{1 / 2} g(u) \\
& \in L^{\infty}\left(R^{+}, V_{-2}\right) .
\end{aligned}
$$

Now, we look for the approximate solutions $u^{n}$ of problem (14) and (15) of the form

$$
u^{n}(t)=\sum_{j=1}^{n} T_{j n}(t) w_{j}
$$

where $A w_{j}=\lambda_{j} w_{j}, j=1,2, \ldots,\left\{w_{j}\right\}$ is an orthonormal basis in $H$, and at the same time an orthogonal one in $V_{2}$, and $T_{j n}(t)=\left(u^{n}, w_{j}\right)$ with

$$
\begin{aligned}
& \left(A^{-1 / 2} u_{t t}^{n}, w_{j}\right)+\left(\left(I+A^{1 / 2}\right) u^{n}, w_{j}\right) \\
& \quad+2 \eta\left(A^{1 / 4} u_{t}^{n}, w_{j}\right)+\left(u_{t}^{n}, w_{j}\right)+\left(g\left(u^{n}\right), w_{j}\right) \\
& \quad=\left(A^{-1 / 2} f, w_{j}\right), \quad t>0, j=1, \ldots, n, \\
& \left(u^{n}(0), u_{t}^{n}(0)\right)=\left(u_{0 n}, u_{1 n}\right) \longrightarrow\left(u_{0}, u_{1}\right) \text { in } E .
\end{aligned}
$$

Obviously, the estimate (38) is valid for $u^{n}$. So we can extract a subsequence, still denoted by $\left\{u^{n}\right\}$, such that

$$
\begin{aligned}
&\left(u^{n}, u_{t}^{n}\right) \longrightarrow\left(u, u_{t}\right) \\
& \text { weakly }^{*} \text { in } L_{\mathrm{loc}}^{\infty}\left(\mathbb{R}^{+} ; E\right) ; \\
& u_{t t}^{n} \longrightarrow u_{t t} \quad \text { weakly }^{*} \text { in } L_{\text {loc }}^{\infty}\left(\mathbb{R}^{+} ; V_{-2}\right) ; \\
&\left(u^{n}(t), u_{t}^{n}(t)\right) \longrightarrow\left(u(t), u_{t}(t)\right) \quad \text { for } t \geq 0 .
\end{aligned}
$$


Applying Lemma 3 to (33), we have

$$
\left(u^{n}, u_{t}^{n}\right) \longrightarrow\left(u, u_{t}\right) \quad \text { in } C_{w}([0, T] ; E) .
$$

Indeed, when $1 \leq p \leq N /(N-2)^{+}$, we have $H_{0}^{1} \hookrightarrow L^{2 p}$; hence

$$
\begin{aligned}
& \int_{0}^{t}\left|\left(g\left(u^{n}\right)-g(u), w_{j}\right)\right| d \tau \\
& \quad \leq C \int_{0}^{t}\left(\left\|u^{n}-u\right\|+\left\|A^{1 / 4} u^{n}-A^{1 / 4} u\right\|\right) d \tau \longrightarrow 0,
\end{aligned}
$$

when $N /(N-2)^{+}<p<(N+2) /(N-2)^{+}, V_{2-\delta} \hookrightarrow L^{2(p+1)}$ and by virtue of the interpolation theorem,

$$
\begin{aligned}
& \int_{0}^{t}\left|\left(g\left(u^{n}\right)-g(u), w_{j}\right)\right| d \tau \leq C \int_{0}^{t}\left(\left\|u^{n}-u\right\|\right. \\
& \left.+\left\|A^{1 / 4}\left(u^{n}-u\right)\right\|^{\theta}\left\|A^{1 / 2}\left(u^{n}-u\right)\right\|^{1-\theta}\right) d \tau \\
& \quad \longrightarrow 0 .
\end{aligned}
$$

Letting $n \rightarrow \infty$ in (41) we see that $u$ is a weak solution of problem (14) and (15), with $\left(u, u_{t}\right) \in C_{w}\left(\mathbb{R}^{+} ; E\right)$.

Integrating (31) over $\left(t_{0}, t\right)$,

$$
\begin{aligned}
& \left(\left\|u_{t}(t)\right\|^{2}+\left\|A^{1 / 4} u(t)\right\|^{2}+\left\|A^{1 / 2} u(t)\right\|^{2}\right) \\
& -\left(\left\|u_{t}\left(t_{0}\right)\right\|^{2}+\left\|A^{1 / 4} u\left(t_{0}\right)\right\|^{2}+\left\|A^{1 / 2} u\left(t_{0}\right)\right\|^{2}\right) \\
& =2 \int_{t_{0}}^{t}(u, f) d t+2 \int_{t_{0}}^{t}\left\|A^{1 / 4} u_{t}\right\|^{2} d t \\
& +2 \int_{t_{0}}^{t}\left(A^{1 / 4} g(u), A^{1 / 4} u_{t}\right) d t \longrightarrow 0, \text { as } t \longrightarrow t_{0} .
\end{aligned}
$$

Obviously,

$$
\begin{aligned}
\left\|\left(u, u_{t}\right)(t)\right\|_{V_{2} \times H}-\left\|\left(u, u_{t}\right)\left(t_{0}\right)\right\|_{V_{2} \times H} \longrightarrow 0, & \\
& t \longrightarrow t_{0} ;
\end{aligned}
$$

we prove that $\left(u, u_{t}\right) \in C\left([0, T], V_{2} \times H\right), u_{t t} \in C\left([0, T], V_{-1}\right)$ and (22).

(ii) Now, we show that $\left(u, u_{t}\right)$ is Lipschitz continuous in the weak space $E$.

In fact, let $u, v$ be two solutions of problem (14) and (15) as shown above corresponding to initial data $u_{0}, u_{1}$ and $v_{0}, v_{1}$, respectively. Then $z=u-v$ solves

$$
\begin{aligned}
& A^{-1 / 2} z_{t t}+\left(I+A^{1 / 2}\right) z+z_{t}+g(u)-g(v)=0, \\
& z(0)=u_{0}-v_{0} \equiv z_{0}, \\
& z_{t}(0)=u_{1}-v_{1} \equiv z_{1} .
\end{aligned}
$$

Using the multiplier $A^{1 / 2} z_{t}$ in (48),

$$
\begin{aligned}
H_{3}\left(z, z_{t}\right)= & \frac{1}{2}\left(\left\|z_{t}\right\|^{2}+\left\|A^{1 / 4} z\right\|^{2}+\left\|A^{1 / 2} z\right\|^{2}\right) \\
& -\left(A^{1 / 2} z_{t}, g(u)-g(v)\right) \\
\leq & \frac{1}{2}\left\|A^{1 / 4} z_{t}\right\|^{2}+C(R)\left\|A^{1 / 2} z\right\|^{2} .
\end{aligned}
$$

We get

$$
\begin{aligned}
& \frac{d}{d t} H_{3}\left(z, z_{t}\right)+\kappa H_{3}\left(z, z_{t}\right) \leq C(R)\|z\|^{2}, \\
& \left\|\left(z, z_{t}\right)(t)\right\|_{E}^{2} \leq C(R, T)\left\|\left(z, z_{t}\right)(0)\right\|_{E}^{2}, \\
& 0 \leq t \leq T,(22) \\
& \left\|\left(z, z_{t}\right)(t)\right\|_{E}^{2} \\
& \leq C(R) e^{-\kappa t}\left\|\left(z, z_{t}\right)(0)\right\|_{E}^{2}+\int_{0}^{t} e^{-\kappa(t-\tau)}\|z(\tau)\|^{2} d \tau,
\end{aligned}
$$

Theorem 1 is proved. Under the assumptions of Theorem 1 , with $1 \leq p<p^{*}, N \leq 5$, we can define the solution operator $S(t): E \rightarrow E$,

$$
\begin{aligned}
& S(t)\left(u_{0}, u_{1}\right)=\left(u(t), u_{t}(t)\right) \\
& \text { for every }\left(u_{0}, u_{1}\right) \in E, t \geq 0,
\end{aligned}
$$

where $u$ is the weak solution of problem (14) and (15). Theorem 1 shows that $\{T(t)\}$ constitutes a semigroup on $E$, which is Lipschitz continuous in $E$.

Theorem 4 (existence of the global attractor). Under the same assumptions of Theorem 1 , with $1 \leq p<p^{*},\{T(t)\}$ has a global attractor $\mathscr{A}$ in $E$ and $\mathscr{A} \subset K$, where $K$ is bounded set in $E_{\sigma}=$ $V_{\sigma+2} \times V_{\sigma}, 0<\sigma<1$.

Proof of Theorem 4. Estimate (38) implies that the ball

$$
B_{0}=\left\{(u, v) \in E \mid\|(u, v)\|_{E}^{2} \leq C_{0}\right\}
$$

is an absorbing set of the semigroup $S(t)$ in $E$. For every bound $B$ in $E$,

$$
\operatorname{dist}\left(S(t) B, B_{0}\right) \leq C(B) e^{-\delta t}, \quad \forall B \subset E .
$$

Let: $S(t)=S_{1}(t)+S_{2}(t)$, where $S_{2}(t): V_{2} \times H \rightarrow V_{2} \times H$, $S_{2}(t)\left(u_{0}, u_{1}\right)=\left(\bar{u}, \bar{u}_{t}\right)$ and

$$
\begin{aligned}
A^{-1 / 2} \bar{u}_{t t}+\left(I+A^{1 / 2}\right) \bar{u}+\bar{u}_{t} & =0 \\
t & >0, \bar{u}(0)=u_{0}, \bar{u}_{t}(0)=u_{1} .
\end{aligned}
$$

It is easy to get

$$
\left\|S_{2}(t)\right\|_{\mathscr{L}(E)} \leq C e^{-\delta t}
$$

$S_{1}(t)=S(t)-S_{2}(t), S_{1}(t): E \rightarrow E, S_{1}(t)\left(u_{0}, u_{1}\right)=\left(\widehat{u}, \widehat{u}_{t}\right)$ solves

$$
\begin{aligned}
A^{-1 / 2} \widehat{u}_{t t}+\left(I+A^{1 / 2}\right) \widehat{u}+\widehat{u}_{t} & =A^{-1 / 2} f-g(u), \\
t & >0, \widehat{u}(0)=0, \widehat{u}_{t}(0)=0,
\end{aligned}
$$

where $u \in C_{b}\left(R^{+}, V_{2}\right)$. 
Let $w=\widehat{u}_{t}$,

$$
\begin{aligned}
A^{-1 / 2} w_{t t}+\left(I+A^{1 / 2}\right) w+w_{t} & =-g^{\prime}(u) u_{t}, \\
w(0) & =0=w_{0}, \\
w_{t}(0) & =f-A^{1 / 2} g\left(u_{0}\right)=w_{1},
\end{aligned}
$$

because $f \in V_{\sigma-2}, A^{1 / 2} g\left(u_{0}\right) \in V_{\sigma-2}, w_{1} \in V_{\sigma-2}, g^{\prime}(u) u_{t} \in$ $C_{b}\left(R^{+}, V_{\sigma-2}\right)$.

Therefore

$$
\left(\widehat{u}_{t}, \widehat{u}_{t t}\right) \in C_{b}\left(R^{+}, V_{\sigma} \times V_{\sigma-2}\right) .
$$

We know $\left(I+A^{1 / 2}\right) \widehat{u}=A^{-1 / 2} f-g(u)-A^{-1 / 2} \widehat{u}_{t t}-\widehat{u}_{t} \in$ $C_{b}\left(R^{+}, V_{\sigma}\right), \widehat{u} \in C_{b}\left(R^{+}, V_{\sigma+2}\right)$ so

$$
\left(\widehat{u}, \widehat{u}_{t}\right) \in C_{b}\left(R^{+}, V_{\sigma+2} \times V_{\sigma}\right) .
$$

Thus $\bigcup_{t \geq 0,\left\|\left(\widehat{u}, \widehat{u}_{t}\right)\right\|_{E_{\sigma}} \leq R}\left(\widehat{u}, \widehat{u}_{t}\right)$ is bounded in $V_{\sigma+2} \times V_{\sigma} . V_{\sigma+2} \times$ $V_{\sigma} \hookrightarrow \hookrightarrow V_{2} \times H$,

$$
K=\bigcup_{t \geq 0,\left\|\left(\widehat{u}, \hat{u}_{t}\right)\right\|_{E_{\sigma}} \leq R}\left(\widehat{u}, \widehat{u}_{t}\right)^{E}
$$

is compact in $E=V_{2} \times H$. For every bounded set $B \subset E$,

$$
\begin{aligned}
\operatorname{dist} & \{S(t) B, K\} \\
= & \sup _{\left(u_{0}, u_{1}\right) \in B} \inf _{\left(\widehat{u}, \hat{u}_{t}\right) \in K} \operatorname{dist}\left\{S(t)\left(u_{0}, u_{1}\right),\left(\widehat{u}, \widehat{u}_{t}\right)\right\} \\
\leq & \sup _{\left(u_{0}, u_{1}\right) \in B}\left\|\left(\bar{u}, \bar{u}_{t}\right)\right\|_{E} \longrightarrow 0 .
\end{aligned}
$$

Therefore $S(t)$ has the global attractor $\mathscr{A}=\omega\left(B_{0}\right)$ and

$$
\operatorname{dist}\{\mathscr{A}, K\}=\operatorname{dist}\{S(t) \mathscr{A}, K\} \longrightarrow 0 \quad t \longrightarrow \infty \text {; }
$$

that is $\mathscr{A} \subset K$. This completes the proof.

\section{Exponential Attractor}

Definition 5. The set $\mathbb{A}_{\exp } \subset E$ is called an exponential attractor for the solution semigroup $S(t)$ of acting on the energy space $E$ if

(i) the set $\mathbb{A}_{\exp }$ is a compact set in $E$;

(ii) $\mathbb{A}_{\exp }$ is forward invariant set; that is, $S(t) \mathscr{A} \subset \mathscr{A}, t \geq 0$;

(iii) $\mathbb{A}_{\exp }$ attracts exponentially the images of all bounded set in $E$; that is,

$$
\operatorname{dist}_{E}\left\{S(t) B, \mathscr{A}_{\exp }\right\} \leq Q\left(\|B\|_{E}\right) e^{-\gamma t} ;
$$

for all bounded set $B \subset E$;

(iv) it has finite fractal dimension in $E$; that is, $\operatorname{dim}_{f}\left\{\mathscr{A}_{\text {exp }}, E\right\}<+\infty$.

From Theorem 1, estimate (38) implies that the ball

$$
B_{R}=\left\{\zeta \in E \mid\|\zeta\|_{E} \leq R\right\}
$$

is an absorbing set of the semigroup $T(t)$ in $E$ for $R>C_{0}$. Without loss of generality we assume that $B_{R}$ is a forward invariant set. Let

$$
\mathscr{B}_{R}=\left[\bigcup_{t \geq t_{0}+1} T(t) B_{R}\right]_{E},
$$

where $t_{0}>0$ is chosen such that $T(t) B_{R} \subset B_{R}$ for $t \geq t_{0}$ and []$_{X}$ stands for the closure in space $X$. Obviously, the set $\mathscr{B}_{R}$ is bounded closed set in $E, T(t) \mathscr{B}_{R} \subset \mathscr{B}_{R}, t \geq 0$, and it is also an absorbing set of $T(t) . \mathscr{B}_{R}$ constitutes a complete metric space (with the $E$ norm) and one sees from (22) that the solution semigroup $T(t)$ is continuous on $\mathscr{B}_{R}$, and the system $\left(T(t), \mathscr{B}_{R}\right)$ constitutes a dissipative dynamical system.

Lemma 6 (see [19]). Let $X$ be a Banach space and $M a$ bounded closed set in X. Assume that the mapping $V: M \rightarrow M$ possesses the following properties:

(i) $V$ is Lipschitz on $M$; that is, there exists $L>0$ such that

$$
\left\|V v_{1}-V v_{2}\right\| \leq L\left\|v_{1}-v_{2}\right\|, \quad v_{1}, v_{2} \in M
$$

(ii) there exist compact seminorms $n_{1}(x)$ and $n_{2}(x)$ on $X$ such that

$$
\begin{aligned}
\left\|V v_{1}-V v_{2}\right\| \leq & \eta\left\|v_{1}-v_{2}\right\| \\
& +K\left[n_{1}\left(v_{1}-v_{2}\right)+n_{2}\left(V v_{1}-V v_{2}\right)\right]
\end{aligned}
$$

for any $v_{1}, v_{2} \in M$, where $0<\eta<1$ and $K>0$ are constants. Then for any $\kappa>0$ and $\delta \in(0,1-\eta)$, there exists a positively invariant compact set $A_{\kappa, \delta} \subset M$ of finite fractal dimension such that

$$
\operatorname{dist}\left(V^{k} M, A_{\kappa, \delta}\right) \leq q^{k}, \quad k=1,2, \ldots,
$$

where $q=\eta+\delta<1$, and

$$
\begin{aligned}
\operatorname{dim}_{f} A_{\kappa, \delta} \leq & \left(\ln \frac{1}{\delta+\eta}\right)^{-1} \\
& \cdot\left(\ln m_{0}\left(\frac{2 K\left(1+L^{2}\right)^{1 / 2}}{\delta}\right)+\kappa\right),
\end{aligned}
$$

where $m_{0}(R)$ is the maximal number of pairs $\left(x_{i}, y_{i}\right)$ in $X \times X$ possessing the properties

$$
\begin{aligned}
\left\|x_{i}\right\|^{2}+\left\|y_{i}\right\|^{2} & \leq R^{2}, \\
n_{1}\left(x_{i}-x_{j}\right)+n_{2}\left(y_{i}-y_{j}\right) & >1,
\end{aligned}
$$

$$
i \neq j
$$

Lemma 7. Let $X, Y$ be the metric spaces and let the mapping $h: X \rightarrow Y$ be $\theta$-Hölder continuous on the set $B \subset X$. Then

$$
\operatorname{dim}_{f}\{h(B), Y\} \leq \frac{1}{\theta} \operatorname{dim}_{f}\{B, X\} .
$$


Theorem 8. Let the assumptions of Theorem 1 be in force, with $1 \leq p<p^{*}$. Then the solution semigroup $T(t)$ has an exponential attractor $\mathscr{A}_{\text {exp }}$ in $E$.

Proof. Define the operator

$$
V^{k}=S(k T): \mathscr{B}_{R} \longrightarrow \mathscr{B}_{R}, \quad k \in \mathbb{Z}^{+} .
$$

We show that the discrete system $\left(V^{k}, \mathscr{B}_{R}\right)$ has an exponential attractor.

Definition 9. We introduce the functional space

$$
\begin{gathered}
W(0, T)=\left\{z \in L^{2}\left(0, T ; V_{2}\right) \mid z_{t}\right. \\
\left.\in L^{2}(0, T ; H),\left\|\xi_{z}\right\|_{W}^{2}<\infty\right\},
\end{gathered}
$$

equipped with the norm

$$
\left\|\xi_{z}\right\|_{W}^{2}=\int_{0}^{T}\left\|\left(z(t), z_{t}(t)\right)\right\|_{E}^{2} d t
$$

and the functional space

$$
H_{T}=E \times W(0, T),
$$

equipped with the usual graph norm; that is

$$
\|U\|_{H_{T}}^{2}=\|\eta\|_{E}^{2}+\left\|\xi_{z}\right\|_{W}^{2}, \quad \forall U=\left(\eta, \xi_{z}\right) \in H_{T} .
$$

Obviously, the spaces $W(0, T)$ and $H_{T}$ are Banach spaces. Let the set

$$
\begin{aligned}
B_{T} & =\left\{\left(\xi_{u}(0), \xi_{u}(t), t \in[0, T]\right) \mid \xi_{u}(0) \in \mathscr{B}_{r}, \xi_{u}(t)\right. \\
& \left.=S(t) \xi_{u}(0)\right\} .
\end{aligned}
$$

Define the operator

$$
\begin{aligned}
\mathbb{V}: B_{T} & \longrightarrow H_{T}, \\
\mathbb{V} U & =\left(S(T) \xi_{u}(0), \xi_{u}(T+\cdot)\right) \\
& =\left(S(T) \xi_{u}(0), S(t+T) \xi_{u}(0), t \in[0, T]\right),
\end{aligned}
$$

where $U=\left(\xi_{u}(0), \xi_{u}(\cdot)\right) \in B_{T}$ and in the following $\xi_{u}(\cdot)$ means $\xi_{u}(t), t \in[0, T]$.

Lemma 10. The set $B_{T}$ is a bounded closed set in $H_{T}$.

Proof. Obviously, $B_{T}$ is bounded in $H_{T}$. For any sequence $\left\{U^{n}\right\} \subset B_{T}$,

$$
U^{n}=\left(\xi_{u^{n}}(0), \xi_{u^{n}}(\cdot)\right) \longrightarrow U=\left(\xi_{u}(0), \xi_{v}(\cdot)\right) \text { in } B_{T} .
$$

Since $\xi_{u^{n}}(0) \in \mathscr{B}_{R}$ and $\mathscr{B}_{R}$ is closed in $E, \xi_{u}(0) \in \mathscr{B}_{0}$. By the Lipschitz continuity of $S(t)$ in $E$,

$$
\begin{aligned}
\left\|\xi_{u^{n}}(t)-\xi_{u}(t)\right\|_{E}^{2} & =\left\|S(t) \xi_{u^{n}}(0)-S(t) \xi_{u}(0)\right\|_{E}^{2} \\
& \leq C(R, T)\left\|\xi_{u^{n}}(0)-\xi_{u}(0)\right\|_{E}^{2} \\
& \longrightarrow 0,
\end{aligned}
$$

so by the uniqueness of the limit; $\xi_{v}(\cdot)=\xi_{u}(t)$, that is, $U=$ $\left(\xi_{u}(0), \xi_{v}(\cdot)\right) \in B_{T}$, where $B_{T}$ is closed in $H_{T}$.
Lemma 10 implies that $B_{T}$ is complete with respect to the topology of $H_{T}$, and the dynamical system $\left(\mathbb{V}^{k}, B_{T}\right)$ constitutes a discrete dissipative dynamical system.

Lemma 11. Under the same assumptions of Theorem 1, then discrete dissipative dynamical system $\left(\mathbb{V}^{k}, B_{T}\right)$ has an exponential attractor $\mathbb{A}$.

Proof. Obviously, $\mathbb{V} B_{T} \subset B_{T}$

$$
\begin{aligned}
\forall U_{1} & =\left(\xi_{u_{1}}(0), \xi_{u_{1}}(\cdot)\right), \\
U_{2} & =\left(\xi_{u_{2}}(0), \xi_{u_{2}}(\cdot)\right), \\
z & =u_{1}-u_{2} ;
\end{aligned}
$$

the inequality (93) holds; then integrating (93) over $(T, 2 T)$ we get

$$
\begin{aligned}
& \int_{T}^{2 T}\left(\|z(t)\|_{V_{2}}^{2}+\left\|z_{t}(t)\right\|_{H}^{2}\right) d t \\
& \leq C \int_{T}^{2 T} e^{-\kappa t} d t\left(\left\|z_{0}\right\|_{V_{2}}^{2}+\left\|z_{1}\right\|_{H}^{2}\right) \\
& \quad+C T \int_{0}^{2 T}\|z(\tau)\|^{2} d \tau .
\end{aligned}
$$

Hence,

$$
\begin{aligned}
\left\|\mathbb{V} U_{1}-\mathbb{V} U_{2}\right\|_{H_{T}}^{2} & \left\|S(T) \xi_{u_{1}}(0)-S(T) \xi_{u_{2}}(0)\right\|_{E}^{2}+\left\|\xi_{z}(t+T)\right\|_{W}^{2} \\
\leq & C e^{-\kappa T}\left\|\xi_{u_{1}}(0)-\xi_{u_{2}}(0)\right\|_{E}^{2} \\
& +C \int_{0}^{T} e^{-\kappa(T-\tau)}\|z(\tau)\|^{2} d \tau+\int_{T}^{2 T}\left\|\xi_{z}(t)\right\|_{E}^{2} d t \\
\leq & \eta_{T}\left\|\xi_{u_{1}}(0)-\xi_{u_{2}}(0)\right\|_{E}^{2}+K_{T} \int_{0}^{2 T}\|z(\tau)\|^{2} d \tau \\
\leq & \eta_{T}\left\|U_{1}-U_{2}\right\|_{H_{T}}^{2} \\
& +K_{T}\left(n_{1}\left(U_{1}-U_{2}\right)+n_{1}\left(\mathbb{V} U_{1}-\mathbb{V} U_{2}\right)\right),
\end{aligned}
$$

where $\xi_{z}(t)=\xi_{u_{1}}(t)-\xi_{u_{2}}(t)$,

$$
\begin{array}{r}
\eta_{T}=2 C e^{-\kappa T}+C \int_{T}^{2 T} e^{-\kappa t} d t, \quad K_{T}=C(T+2), \\
n_{1}(U)=\int_{0}^{T}\|u(t)\|^{2} d t, \quad U=\left(\xi_{u}(0), \xi_{u}(\cdot)\right) \in B_{T} .
\end{array}
$$

It follows from (83) that

$$
\left\|\mathbb{V} U_{1}-\mathbb{V} U_{2}\right\|_{H_{T}}^{2} \leq a_{T}\left\|U_{1}-U_{2}\right\|_{H_{T}}^{2},
$$

where $a_{T}=\eta_{T}+C K_{T} \int_{0}^{2 T} e^{\kappa t} d t$. Since $W(0, T) \hookrightarrow \hookrightarrow$ $L^{2}(0, T ; H)$, the seminorm $n_{1}(U)$ is compact in $H_{T}$. Taking 
$T: 0<\eta_{T}<1$ and making use of Lemma 6, we get the conclusion of Lemma 11. That is, the discrete dynamical system $\left(V^{k}, M\right)$ possesses an exponential attractor $A_{\kappa, \delta}$. Define the project operator

$$
\begin{aligned}
\Pi: B_{T} & \longrightarrow \mathscr{B}_{R}, \\
\Pi U & =\xi_{u}(0), \\
U & =\left(\xi_{u}(0), \xi_{u}(\cdot)\right) \in B_{T} .
\end{aligned}
$$

Lemma 12. $A=\Pi A$ is an exponential attractor of the discrete dynamical system $\left(V^{k}, \mathscr{B}_{R}\right)$.

Proof. (1) $A$ is compact because $A$ is the image of the compact set $\mathbb{A}$ under the continuous mapping $\Pi$.

(2) $\mathbb{V}^{k} A \subset A$; we have $\mathbb{V}^{k} \mathbb{A} \subset \mathbb{A}$; thus $V^{k} A=\Pi \mathbb{V}^{k} \mathbb{A} \subset$ $\Pi \mathbb{A}=A$.

(3) Obviously,

$$
\begin{aligned}
\operatorname{dist}_{E}\left\{V^{k} \mathscr{B}_{R}, A\right\} \leq \operatorname{dist}_{H_{T}}\left\{\mathbb{V}^{k} B_{T}, \mathbb{A}\right\} \leq C q^{k}, & \\
& 0<q<1 ;
\end{aligned}
$$

for some $0<q<1$ (see Lemma 11).

(4) $\operatorname{dim}_{f}\{A, E\} \leq \operatorname{dim}_{f}\left\{\mathbb{A}, H_{T}\right\}<\infty$.

Hence, $A$ is a desired exponential attractor. Lemma 12 is proved.

Let

$$
\mathbb{A}_{\exp }=\bigcup_{0 \leq t \leq S} S(t) A
$$

By the method used in [11], one easily knows that $\mathbb{A}_{\exp }$ is an exponential attractor of $\left(S(t), \mathscr{B}_{R}\right)$, with $E$ topology. So by the definition of the exponential attractor, there exists a constant $\kappa>0$, such that

$$
\operatorname{dist}_{E}\left\{S(t) \mathscr{B}_{R}, A_{\exp }\right\} \leq C e^{-\kappa t}, \quad t>0 .
$$

Since the set $\mathbb{A}_{\exp } \subset \mathscr{B}_{0}$ is bounded in $E$, we claim that $\mathbb{A}_{\text {exp }}$ is an exponential attractor of the system $(S(t), E)$. Indeed, (i) obviously, $\mathbb{A}_{\exp }$ is forward invariant; (ii) define the project operator

$$
\begin{aligned}
& F:[0, T] \times A \longrightarrow \mathscr{B}_{R}, \\
& F\left(t, \xi_{u}\right)=\xi_{u}(t), \quad \xi_{u}(t) \in \mathscr{B}_{R}, \quad t \in[0, T], \\
& \left\|F\left(t_{1}, \xi_{u}\right)-F\left(t_{2}, \xi_{u}\right)\right\|_{E} \\
& \quad \leq C\left(\int_{t_{1}}^{t_{2}}\left\|\xi_{u}^{\prime}(\tau)\right\|_{E}^{2} d \tau\right)^{1 / 2}\left|t_{1}-t_{2}\right|^{1 / 2} \\
& \quad \leq C\left|t_{2}-t_{1}\right|^{1 / 2}, \\
& \left\|F\left(t, \xi_{u_{1}}\right)-F\left(t, \xi_{u_{2}}\right)\right\|_{E_{1}} \leq C(R) e^{\kappa T}\left\|\xi_{u_{1}}-\xi_{u_{2}}\right\|_{E}
\end{aligned}
$$

for any $\xi_{u}, \xi_{u_{1}}, \xi_{u_{2}} \in \mathscr{B}_{R}, t, t_{1}, t_{2} \in[0, T]$, which imply that the mapping $F$ is $1 / 2$-Hölder continuous. Therefore, $\mathbb{A}_{\exp }=$ $F\{[0, T] \times A\}$ (the image of $[0, T] \times A)$ is compact in $E$. (iii) Consider the following:

$$
\begin{aligned}
\operatorname{dim}_{f}\left\{\mathbb{A}_{\text {exp }}, E\right\} & \leq 2 \operatorname{dim}_{f}\left\{[0, S] \times A, \mathbb{R}^{+} \times E\right\} \\
& \leq 2\left(1+\operatorname{dim}_{f}\{A, E\}\right)<\infty
\end{aligned}
$$

(iv) For any $t \in \mathbb{R}^{+}$, there exists a $k \in \mathbb{N}^{+}$, such that $S(t) \mathscr{B}_{R} \subset$ $T(k T) \mathscr{B}_{R}$ as $t \in(k T,(k+1) T]$. On account of $A=S(0) A \subset$ $\mathbb{A}_{\text {exp }}$,

$$
\begin{aligned}
\operatorname{dist}_{E}\left\{S(t) \mathscr{B}_{R}, \mathbb{A}_{\exp }\right\} \leq \operatorname{dist}_{E}\left\{S(k T) \mathscr{B}_{R}, A\right\} \\
\leq \sup _{\xi_{u} \in \mathscr{B}_{R}} \inf _{\xi_{v} \in A}\left\|S(t) \xi_{u}-\xi_{v}\right\|_{E}^{1 / 2} \leq C q^{k / 2} \leq C e^{-\kappa t / 2} .
\end{aligned}
$$

Therefore, Theorem 8 is proved.

\section{Global and Exponential Attractor in Nonsupercritical Case}

Theorem 13. Let the assumptions of Theorem 1 be in force, with $1 \leq p \leq \widetilde{p}=N /(N-2)^{+}$. Then problem (14)(15) admits a unique weak solution $u$, with $\left(u, u_{t}\right) \in C_{b}\left(\mathbb{R}^{+}\right.$, $\left.E_{1}\right) \equiv L^{\infty}\left(\mathbb{R}^{+}, E_{1}\right) \cap C\left(\mathbb{R}^{+}, E_{1}\right)$, and the solution is Lipschitz continuous in $E_{1}=V_{1} \times H$; that is,

$$
\left\|\left(z(t), z_{t}(t)\right)\right\|_{E_{1}}^{2} \leq C e^{k t}\left\|\left(z(0), z_{t}(0)\right)\right\|_{E_{1}}^{2}, \quad t \geq 0,
$$

for some $C, k>0$, where $z=u-v, u$ and $v$ are, respectively, the weak solutions of (14) corresponding to initial data $\left(u_{0}, u_{1}\right)$ and $\left(v_{0}, v_{1}\right)$.

Proof. The existence of the weak solutions can be easily proved by the same way of Theorem 1 . So we only prove (93) here. Taking $H$-inner product by $z_{t}$ in (36), we have

$$
\begin{aligned}
& \frac{1}{2} \frac{d}{d t}\left(\left\|A^{-1 / 4} z_{t}\right\|^{2}+\|z\|^{2}+\left\|A^{1 / 4} z\right\|^{2}\right)+\left\|z_{t}\right\|^{2} \\
& \quad=-\left(g(u)-g(v), z_{t}\right) \\
& \quad \leq C\left(1+\|u\|_{2 p}^{p-1}+\|v\|_{2 p}^{p-1}\right)\|z\|_{2 p}\left\|z_{t}\right\| \\
& \quad \leq \frac{1}{2}\left\|z_{t}\right\|^{2}+C\left\|A^{1 / 4} z\right\|^{2} .
\end{aligned}
$$

Applying the Gronwall inequality to (94) we obtain (93).

Remark 14. (i) When $1 \leq p \leq \widetilde{p}$, by (93), define the continuous semigroup

$$
\begin{aligned}
& S(t): E_{1} \longrightarrow E_{1}, \\
& \quad S(t) \varphi_{0}=\varphi_{u}(t)=\left(u(t), u_{t}(t)\right),
\end{aligned}
$$

where $\varphi_{u}=\left(u, u_{t}\right) \in C_{b}\left(\mathbb{R}^{+}, E_{1}\right)$ as shown in Theorem 13 .

(ii) It follows from Theorem 8 and Remark 14 that the dynamical system $\left(S(t), E_{1}\right)$ is dissipative; that is, it has a bounded absorbing set $\mathscr{B}_{R}$. Without loss of generality we assume that $\mathscr{B}_{R}$ is positive invariant; that is, $S(t) \mathscr{B}_{R} \subset \mathscr{B}_{R}$ for $t \geq 0$. 
Theorem 15. Let the assumptions of Theorem 13 be in force, especially when $p=\tilde{p}, g \in C^{2}(\mathbb{R})$,

$$
\left|g^{\prime \prime}(s)\right| \leq C\left(1+|s|^{p-2}\right), \quad s \in \mathbb{R} \text { with } p \geq 2 .
$$

Then the following conclusions are valid.

(i) The solution semigroup $S(t)$ possesses in $E_{1}$ a compact global attractor $\mathscr{A}$, which has finite fractal dimension.

(ii) Any full trajectory $v=\left\{\varphi_{u}(t)=\left(u(t), u_{t}(t)\right) \mid t \in \mathbb{R}\right\} \subset$ $\mathscr{A}$ possesses the property

$$
\left(u, u_{t}, u_{t t}\right) \in L^{\infty}\left(\mathbb{R} ; V_{2} \times V_{1} \times H\right),
$$

and there exists constant $R>0$ such that

$$
\sup _{\nu \mathscr{A}} \sup _{t \in \mathbb{R}}\left(\|u(t)\|_{V_{2}}^{2}+\left\|u_{t}(t)\right\|_{V_{1}}^{2}+\left\|u_{t t}(t)\right\|^{2}\right) \leq R^{2} .
$$

(iii) The global attractor $\mathscr{A}$ consists of full trajectory $v=\left\{\varphi_{u}(t) \mid\right.$ $t \in \mathbb{R}\}$ such that

$$
\begin{aligned}
& \lim _{t \rightarrow-\infty} \operatorname{dist}_{E_{1}}\left\{\varphi_{u}(t), \mathcal{N}\right\}=0, \\
& \lim _{t \rightarrow+\infty} \operatorname{dist}_{E_{1}}\left\{\varphi_{u}(t), \mathcal{N}\right\}=0,
\end{aligned}
$$

where $\mathcal{N}$ is the set of all fixed points of $T(t)$; that is,

$$
\mathcal{N}=\left\{(u, 0) \in E_{1} \mid\left(I+A^{1 / 2}\right) u+g(u)=A^{-1 / 2} f\right\} .
$$

Furthermore, for any $\zeta \in E_{1}$,

$$
\lim _{t \rightarrow+\infty} \operatorname{dist}_{E_{1}}\{S(t) \zeta, \mathcal{N}\}=0 .
$$

(iv) The semigroup $S(t)$ has in $E_{1}$ an exponential attractor.

Lemma 16. Let $y: \mathbb{R}^{+} \rightarrow \mathbb{R}^{+}$be an absolutely continuous function satisfying

$$
\frac{d}{d t} y(t)+2 \epsilon y(t) \leq h(t) y(t)+z(t), \quad t>0,
$$

where $\epsilon>0, z \in L_{\text {loc }}^{1}\left(\mathbb{R}^{+}\right), \int_{s}^{t} h(\tau) d \tau \leq \epsilon(t-s)+m$ fort $\geq s \geq 0$ and some $m>0$. Then

$$
y(t) \leq e^{m}\left(y(0) e^{-\epsilon t}+\int_{0}^{t}|z(\tau)| e^{-\epsilon(t-\tau)} d \tau\right),
$$

$$
t>0 \text {. }
$$

Lemma 17 (quasi-stability). Let the assumptions of Theorem 13 be valid and let $u, v$ be the solutions of problem (14)-(15) with initial data in $\mathscr{B}_{R}$. Then $z=u-v$ satisfies the relation

$$
\begin{aligned}
\left\|\left(z(t), z_{t}(t)\right)\right\|_{E_{1}}^{2} \leq & C\left\|\left(z(0), z_{t}(0)\right)\right\|_{E_{1}}^{2} e^{-\kappa t} \\
& +K \sup _{0 \leq s \leq t}\|z(s)\|^{2}
\end{aligned}
$$

for some constants $C, K>0$.
Proof. (i) When $1 \leq p<\widetilde{p}$, taking $H$-inner product by $z_{t}+\epsilon z$ in (48), with $\eta=0$, we get

$$
\begin{gathered}
\frac{d}{d t} H_{4}\left(z, z_{t}\right)+\left\|z_{t}\right\|^{2}-\epsilon\left\|A^{-1 / 4} z_{t}\right\|^{2} \\
+\epsilon\left(\|z\|^{2}+\left\|A^{1 / 4} z\right\|^{2}\right) \\
=-\left(g(u)-g(v), z_{t}+\epsilon z\right),
\end{gathered}
$$

where

$$
\begin{aligned}
& H_{4}\left(z, z_{t}\right)=\frac{1}{2}\left(\left\|A^{-1 / 4} z_{t}\right\|^{2}+(1+\epsilon)\|z\|^{2}+\left\|A^{1 / 4} z\right\|^{2}\right. \\
& \left.\quad+2 \epsilon\left(A^{-1 / 2} z_{t}, z\right)\right) \sim\left\|\left(z, z_{t}\right)\right\|_{E_{1}}^{2}
\end{aligned}
$$

for $\epsilon>0$ suitably small. On account of $p<\widetilde{p}, V_{1-\delta} \hookrightarrow L^{2 p}$ for $\delta: 0<\delta \ll 1$ and the interpolation theorem we have the control

$$
\begin{aligned}
& \left|\left(g(u)-g(v), z_{t}+\epsilon z\right)\right| \leq C\left(1+\|u\|_{2 p}^{p-1}+\|v\|_{2 p}^{p-1}\right) \\
& \cdot\left(\|z\|_{2 p}\left\|z_{t}\right\|+\epsilon\|z\|_{2 p}\|z\|\right) \leq C\|z\|_{V_{1-\delta}} \\
& \cdot\left(\left\|z_{t}\right\|+\epsilon\|z\|\right) \leq \frac{1}{2}\left\|z_{t}\right\|^{2}+\frac{\epsilon}{2}\left\|A^{1 / 4} z\right\|^{2}+C\|z\|^{2} .
\end{aligned}
$$

Therefore, there exists constant $\kappa>0$ such that

$$
\begin{aligned}
& \frac{d}{d t} H_{4}\left(z, z_{t}\right)+\kappa H_{4}\left(z, z_{t}\right) \leq C\|z\|^{2}, \\
& \left\|\left(z(t), z_{t}(t)\right)\right\|_{E_{1}}^{2} \\
& \leq C\left\|\left(z(0), z_{t}(0)\right)\right\|_{E_{1}}^{2} e^{-\kappa t} \\
& \quad+C \int_{0}^{t} e^{-\kappa(t-\tau)}\|z(\tau)\|^{2} d \tau \\
& \leq C\left\|\left(z(0), z_{t}(0)\right)\right\|_{E_{1}}^{2} e^{-\kappa t}+K \sup _{0 \leq \tau \leq t}\|z(\tau)\|^{2},
\end{aligned}
$$

where $K=C / \kappa$.

(ii) When $p=\widetilde{p}$, rewrite (105) in the form

$$
\begin{gathered}
\frac{d}{d t}\left(H_{4}\left(z, z_{t}\right)+\frac{1}{2}(g(u)-g(v), z)\right)+\left\|z_{t}\right\|^{2} \\
-\epsilon\left\|A^{-1 / 4} z_{t}\right\|^{2}+\epsilon\left(\|z\|^{2}+\left\|A^{1 / 4} z\right\|^{2}\right) \\
+\epsilon(g(u)-g(v), z)=\frac{1}{2}\left(\tilde{g}(u, v), z^{2}\right),
\end{gathered}
$$

where

$$
\begin{aligned}
& \tilde{g}(u, v) \\
& \quad=\int_{0}^{1} g^{\prime \prime}(\lambda u+(1-\lambda) v)\left(\lambda u_{t}+(1-\lambda) v_{t}\right) d \lambda .
\end{aligned}
$$


Since $V_{1} \hookrightarrow L^{2 p}$, there exists constant $l>0$ such that

$$
\begin{aligned}
& |(g(u)-g(v), z)| \\
& \quad \leq C\left(1+\|u\|_{2 p}^{p-1}+\|v\|_{2 p}^{p-1}\right)\|z\|_{2 p}\|z\| \leq C\|z\|_{V_{1}}\|z\| \\
& \quad \leq \frac{1}{2}\|z\|_{V_{1}}^{2}+l\|z\|^{2},
\end{aligned}
$$

which means

$$
\begin{aligned}
& (g(u)-g(v), z)+l\|z\|^{2} \geq-\frac{1}{2}\|z\|_{V_{1}}^{2}, \\
& \left|\left(\tilde{g}(u, v), z^{2}\right)\right| \\
& \quad \leq C\left(1+\|u\|_{2 p}^{p-2}+\|v\|_{2 p}^{p-2}\right)\left(\left\|u_{t}\right\|+\left\|v_{t}\right\|\right)\|z\|_{2 p}^{2} \\
& \quad \leq C\left(\left\|u_{t}\right\|+\left\|v_{t}\right\|\right)\|z\|_{V_{1}}^{2} .
\end{aligned}
$$

We infer from (109) that

$$
\begin{aligned}
& \frac{d}{d t} H_{5}\left(z, z_{t}\right)+\frac{1}{2}\left\|z_{t}\right\|^{2}+K_{5}\left(z, z_{t}\right) \\
& \quad \leq C\left(\left\|u_{t}\right\|+\left\|v_{t}\right\|\right)\|z\|_{V_{1}}^{2}+l\left(z, z_{t}\right) \\
& \quad \leq C\left(\left\|u_{t}\right\|+\left\|v_{t}\right\|\right) H_{5}\left(z, z_{t}\right)+\frac{1}{2}\left\|z_{t}\right\|^{2}+l^{2}\|z\|^{2},
\end{aligned}
$$

where

$$
\begin{aligned}
H_{5} & \left(z, z_{t}\right) \\
= & H_{4}\left(z, z_{t}\right)+\frac{1}{2}\left((g(u)-g(v), z)+l\|z\|^{2}\right) \\
\sim & \left\|z_{t}\right\|^{2}+\left\|A^{1 / 4} z\right\|^{2}, \\
K_{5} & \left(z, z_{t}\right) \\
= & \left(\frac{1}{2}-\epsilon-\frac{\epsilon}{\sqrt{\lambda_{1}}}\right)\left\|z_{t}\right\|^{2} \\
\quad & +\epsilon\left(\|z\|^{2}+\left\|A^{1 / 4} z\right\|^{2}+(g(u)-g(v), z)\right) \\
\geq & 2 \kappa H_{5}\left(z, z_{t}\right)-l \epsilon\|z\|^{2}
\end{aligned}
$$

for $\epsilon>0$ suitably small. Inserting (114) into (113), we get

$$
\begin{aligned}
& \frac{d}{d t} H_{5}\left(z, z_{t}\right)+2 \kappa H_{5}\left(z, z_{t}\right) \\
& \quad \leq C\left(\left\|u_{t}\right\|+\left\|v_{t}\right\|\right) H_{5}\left(z, z_{t}\right)+l^{2}\|z\|^{2} .
\end{aligned}
$$

There exists $m>0$ such that

$$
\begin{aligned}
& C \int_{s}^{t}\left(\left\|u_{t}(\tau)\right\|+\left\|v_{t}(\tau)\right\|\right) d \tau \\
& \quad \leq C\left(\int_{s}^{t}\left(\left\|u_{t}(\tau)\right\|^{2}+\left\|v_{t}(\tau)\right\|^{2}\right) d \tau\right)^{1 / 2}(t-s)^{1 / 2} \\
& \quad \leq \kappa(t-s)+m, \quad \text { for } t \geq s \geq 0 .
\end{aligned}
$$

Applying Lemma 16 to (115), we get

$$
\begin{aligned}
& \left\|\left(z(t), z_{t}(t)\right)\right\|_{E_{1}}^{2} \leq C e^{m}\left(\left\|\left(z(0), z_{t}(0)\right)\right\|_{E_{1}}^{2} e^{-\kappa t}\right. \\
& \left.\quad+l^{2} \int_{0}^{t} e^{-\kappa(t-\tau)}\|z(\tau)\|^{2} d \tau\right) \\
& \quad \leq C e^{m}\left\|\left(z(0), z_{t}(0)\right)\right\|_{E_{1}}^{2} e^{-\kappa t}+K \sup _{0 \leq \tau \leq t}\|z(\tau)\|^{2},
\end{aligned}
$$

where $K=C e^{m} l^{2} / \kappa$. Lemma 17 is proved.

Proof of Theorem 15. The estimates (93) and (104) show that the dissipative system $\left(T(t), E_{1}\right)$ is quasi-stable on the absorbing set $\mathscr{B}_{R}$, so the conclusions (i) and (ii) follow directly from the standard theory on global attractor (cf. Theorems 7.9.4-7.9.6 and 7.9.8 in [30]).

The energy equality holds and shows that $H\left(u, u_{t}\right)$ is a strictly Lyapunov function on $E_{1}$, so the dynamical system $\left(T(t), E_{1}\right)$ is gradient, and by conclusion (ii), it has a compact global attractor. Therefore, the conclusion (iii) of Theorem 4 holds (cf. Theorems 2.28 and 2.31 in [20]).

We see from the conclusion (ii) that the global attractor $\mathscr{A}$ is included and bounded in $E_{2}=V_{2} \times V_{1}$. Let $\mathscr{D}$ be the closure of the 1-neighborhood of $\mathscr{A}$ in $E_{2}$; that is,

$$
\mathscr{D}=\left[\left\{\zeta \in E_{2} \mid \operatorname{dist}_{E_{2}}\{\zeta, \mathscr{A}\} \leq 1\right\}\right]_{E_{1}} .
$$

Then $\mathscr{D}$ is bounded in $E_{2}$ and closed in $E_{1}$, and it is an absorbing set of $T(t)$; without loss of generality we assume that $T(t) \mathscr{D} \subset \mathscr{D}, t \geq 0$. By Lemma 17, $T(t)$ is quasi-stable on $\mathscr{D}$. For every $\varphi_{0} \in \mathscr{D}, \varphi(t)=S(t) \varphi_{0}=\left(u(t), u_{t}(t)\right) \in \mathscr{D}$ and by (14), $\left\|u_{t t}\right\| \leq C(\mathscr{D})$,

$$
\begin{aligned}
& \left\|T\left(t_{2}\right) \varphi_{0}-T\left(t_{1}\right) \varphi_{0}\right\|_{E_{1}} \leq \int_{t_{1}}^{t_{2}}\left\|\varphi^{\prime}(t)\right\|_{E_{1}} d t \\
& \quad \leq C(\mathscr{D})\left|t_{2}-t_{1}\right| .
\end{aligned}
$$

So $T(t)$ has in $E_{1}$ an exponential attractor (cf. Theorem 7.9.9 in [30]). Theorem 15 is proved.

Remark 18. Comparing Theorem 8 with Theorem 2.2 in [17] one finds that the critical case $p=\widetilde{p}$ is solved in natural energy space $E$, the restriction $N \leq 5$ is removed in the subcritical case $p<\tilde{p}$, the higher regularity of the global attractor is obtained, and the exponential attractor is established in $E_{1}$.

\section{Competing Interests}

The authors declare that there are no competing interests regarding the publication of this paper.

\section{References}

[1] J. Boussinesq, “Theorie des ondes et des temous qui se prepatent le long d'un canal rectangularly horizontal, en communicant au liquids contend dans ce canal des vitesses sunsiblemend parielles de la surface an fond," Journal de Mathématiques Pures et Appliquées, vol. 17, pp. 55-108, 1872. 
[2] M. S. Bruzón and M. L. Gandarias, "Similarity reductions of a generalized double dispersion equation," Proceedings in Applied Mathematics and Mechanics, vol. 8, no. 1, pp. 10587-10588, 2008.

[3] M. S. Bruzón and M. L. Gandarias, "Travelling wave solutions for a generalized double dispersion equation," Nonlinear Analysis: Theory, Methods \& Applications, vol. 71, no. 12, pp. e2109e2117, 2009.

[4] G.-W. Chen, Y.-P. Wang, and S.-B. Wang, "Initial boundary value problem of the generalized cubic double dispersion equation," Journal of Mathematical Analysis and Applications, vol. 299, no. 2, pp. 563-577, 2004.

[5] G.-W. Chen and H.-X. Xue, "Periodic boundary value problem and Cauchy problem of the generalized cubic double dispersion equation," Acta Mathematica Scientia B, vol. 28, no. 3, pp. 573587, 2008.

[6] M. Kato, Y.-Z. Wang, and S. Kawashima, "Asymptotic behavior of solutions to the generalized cubic double dispersion equation in one space dimension," Kinetic and Related Models, vol. 6, no. 4, pp. 969-987, 2013.

[7] S. Kawashima and Y.-Z. Wang, "Global existence and asymptotic behavior of solutions to the generalized cubic double dispersion equation," Analysis and Applications, vol. 13, no. 3, p. 233, 2015.

[8] J. L. Bona and R. L. Sachs, "Global existence of smooth solutions and stability of solitary waves for a generalized Boussinesq equation," Communications in Mathematical Physics, vol. 118, no. 1, pp. 15-29, 1988.

[9] Y.-C. Liu and R.-Z. Xu, "Potential well method for initial boundary value problem of the generalized double dispersion equations," Communications on Pure and Applied Analysis, vol. 7, no. 1, pp. 63-81, 2008.

[10] A. M. Samsonov, "On some exact travelling wave solutions for nonlinear hyperbolic equation," in Pitman Research Notes in Mathematics Series, vol. 227, pp. 123-132, Longman, Harlow, UK, 1993.

[11] S.-B. Wang and G.-W. Chen, "Cauchy problem of the generalized double dispersion equation," Nonlinear Analysis: Theory, Methods \& Applications, vol. 64, no. 1, pp. 159-173, 2006.

[12] S.-B. Wang and F. Da, "On the asymptotic behaviour of solution for the generalized double dispersion equation," Applicable Analysis, vol. 92, no. 6, pp. 1179-1193, 2013.

[13] R.-Z. Xu and Y.-C. Liu, "Global existence and nonexistence of solution for Cauchy problem of multidimensional double dispersion equations," Journal of Mathematical Analysis and Applications, vol. 359, no. 2, pp. 739-751, 2009.

[14] R. Z. Xu, Y. C. Liu, and T. Yu, "Global existence of solution for Cauchy problem of multidimensional generalized double dispersion equations," Nonlinear Analysis. Theory, Methods \& Applications, vol. 71, no. 10, pp. 4977-4983, 2009.

[15] Z.-D. Dai and B.-L. Guo, "Long time behavior of nonlinear strain waves in elastic waveguides," Journal of Partial Differential Equations, vol. 12, no. 4, pp. 301-312, 1999.

[16] Z.-D. Dai and B.-L. Guo, "Global attractor of nonlinear strain waves in elastic waveguides," Acta Mathematica Scientia, Series B, vol. 20, no. 3, pp. 322-334, 2000.

[17] Z.-J. Yang, "Global attractor for a nonlinear wave equation arising in elastic waveguide model," Nonlinear Analysis: Theory, Methods \& Applications, vol. 70, no. 5, pp. 2132-2142, 2009.

[18] Z. Yang, "A global attractor for the elastic waveguide model in $\mathbb{R}^{N}$," Nonlinear Analysis: Theory, Methods \& Applications, vol. 74, no. 17, pp. 6640-6661, 2011.
[19] Z.-J. Yang and K. Li, "Longtime dynamics for an elastic waveguide model," Discrete and Continuous Dynamical SystemsSeries S, pp. 797-806, 2013.

[20] I. Chueshov and I. Lasiecka, "Long-time behavior of second order evolution equations with nonlinear damping," in Memories of AMS, vol. 195, American Mathematical Society, Providence, RI, USA, 2008.

[21] I. Chueshov and I. Lasiecka, "Existence, uniqueness of weak solutions and global attractors for a class of nonlinear 2D Kirchhoff-Boussinesq models," Discrete and Continuous Dynamical Systems, vol. 15, no. 3, pp. 777-809, 2006.

[22] P. Galenko and D. Jou, "Diffuse-interface model for rapid phase transformations in nonequilibrium systems," Physical Review E, vol. 71, no. 4, Article ID 046125, 2005.

[23] P. Galenko and V. Lebedev, "Analysis of the dispersion relation in spinodal decomposition of a binary system," Philosophical Magazine Letters, vol. 87, no. 11, pp. 821-827, 2007.

[24] P. Galenko and V. Lebedev, "Non-equilibrium effects in spinodal decomposition of a binary system," Physics Letters A, vol. 372, no. 7, pp. 985-989, 2008.

[25] M. Grasselli, G. Schimperna, and S. Zelik, "On the 2D CahnHilliard equation with inertial term," Communications in Partial Differential Equations, vol. 34, no. 1-3, pp. 137-170, 2009.

[26] M. Grasselli, G. Schimperna, A. Segatti, and S. Zelik, "On the 3D Cahn-Hilliard equation with inertial term," Journal of Evolution Equations, vol. 9, no. 2, pp. 371-404, 2009.

[27] M. Grasselli, G. Schimperna, and S. Zelik, “Trajectory and smooth attractors for Cahn-Hilliard equations with inertial term," Nonlinearity, vol. 23, no. 3, pp. 707-737, 2010.

[28] Z.-J. Yang and G.-W. Chen, "Blowup of solutions for a class of generalized Boussinesq equations," Acta Mathematica Scientia, vol. 16, pp. 31-40, 1996.

[29] J. Simon, "Compact sets in the space $L^{p}(\mathrm{O}, T ; B)$," Annali di Matematica Pura ed Applicata, vol. 146, no. 1, pp. 65-96, 1986.

[30] I. Chueshov and I. Lasiecka, Von Karman Evolution Equations, Springer, New York, NY, USA, 2010. 


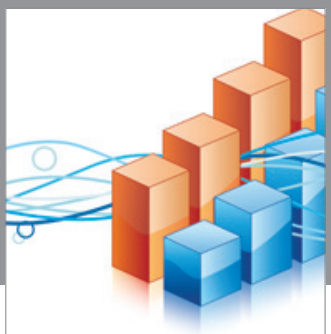

Advances in

Operations Research

vatem alat4

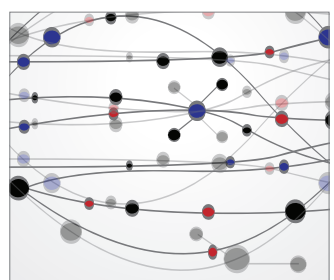

\section{The Scientific} World Journal
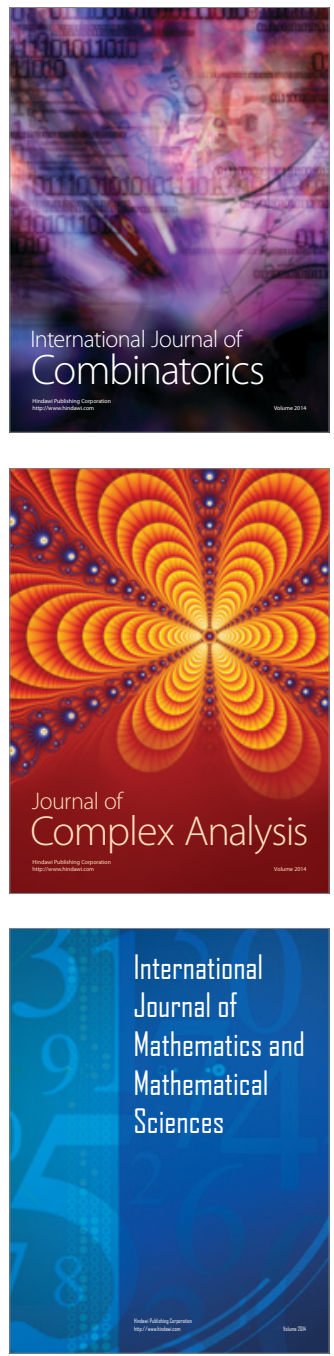
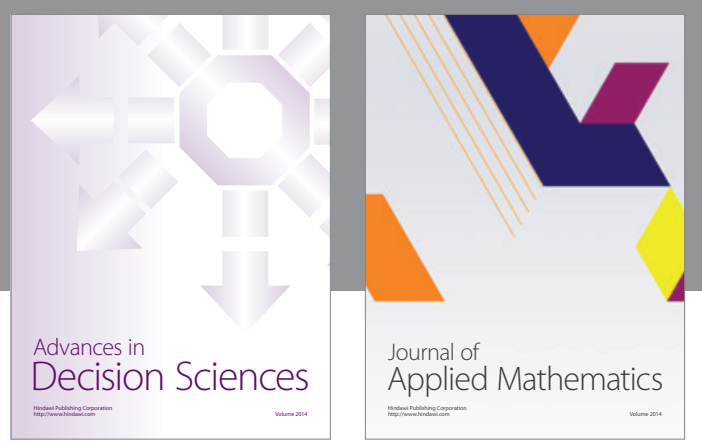

Algebra

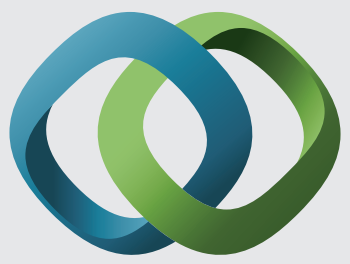

\section{Hindawi}

Submit your manuscripts at

http://www.hindawi.com
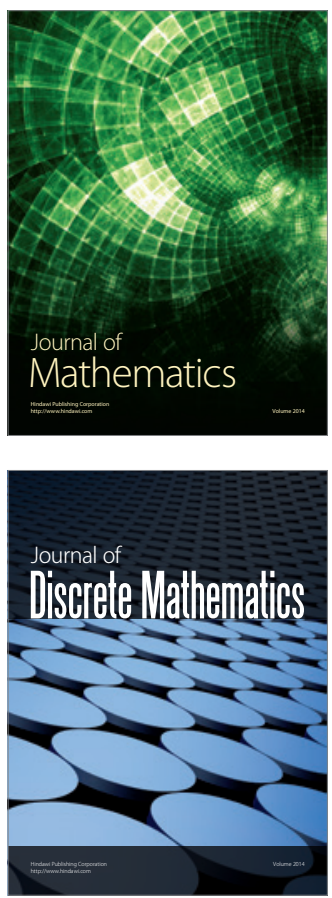

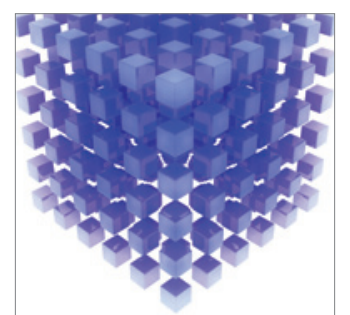

Mathematical Problems in Engineering
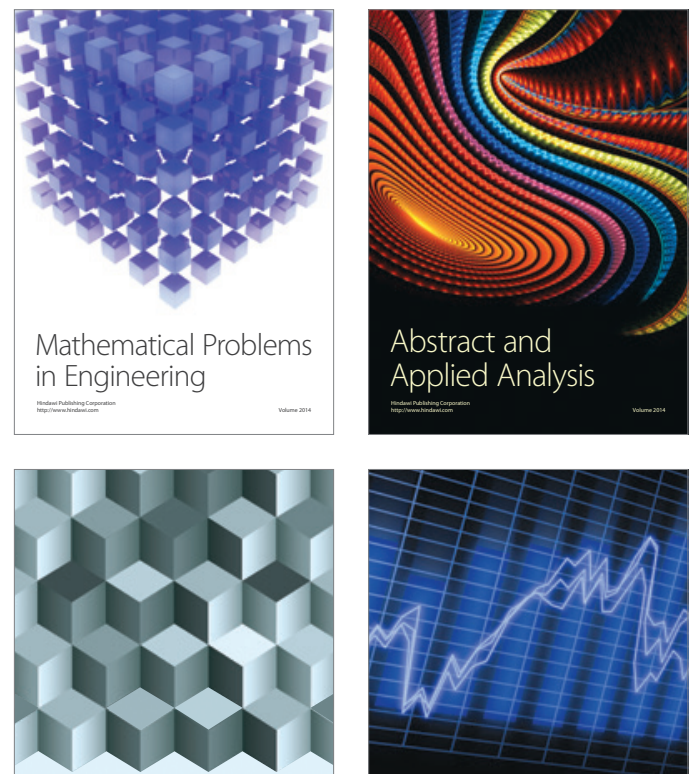

Journal of

Function Spaces

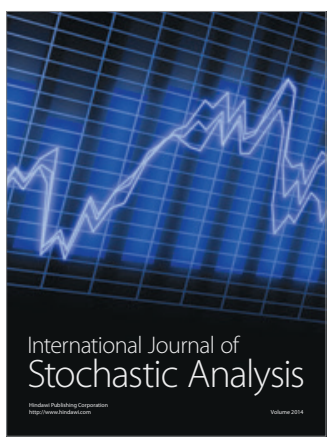

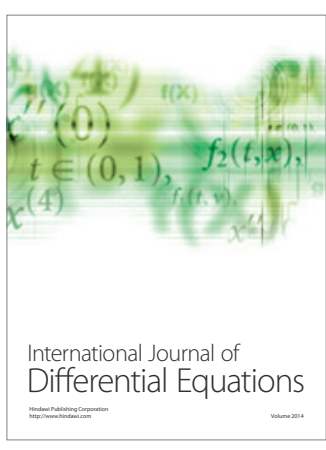
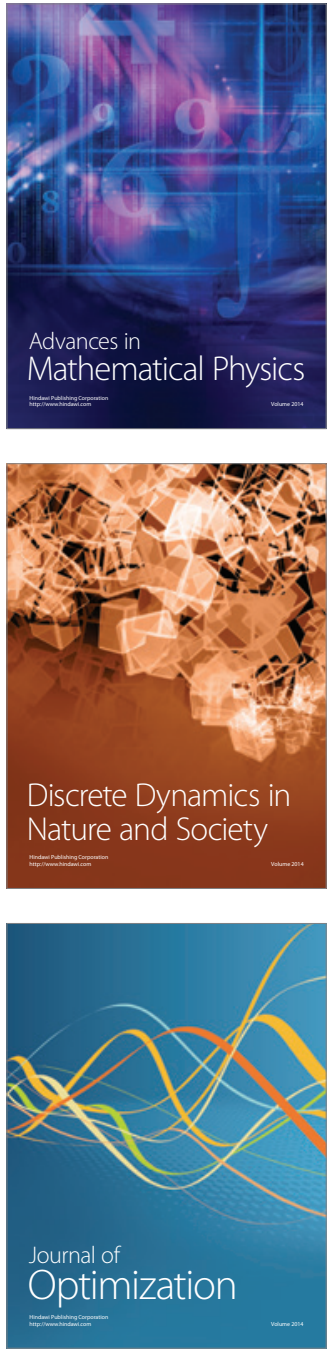